\title{
Numerical study of a three swirling jets system impacting a plane plate
}

\author{
Amar Zerrouta ${ }^{a}$, Ali Khelil and Larbi Loukarfi \\ Control Laboratory, Test, Measurement and Mechanical Simulation, University of Chlef, Algeria, BP 151, \\ 02000 Chlef, Algeria
}

Received 17 March 2015, Accepted 20 January 2016

\begin{abstract}
This investigation deals with a numerical simulation of a multiple swirling jets system impacting a plane plate. The studied configuration composed of three diffusers arranged in line, with diameter $D$ and the gap between the axes of their centers are fixed to $2 D$. The height of impact is fixed to $4 D$, impacting the plate perpendicularly. The swirling jet is generated by swirler system composed of 12 vanes inclined by the angle of $60^{\circ}$ relative to the vertical position just outside of the diffuser. The velocity and the temperature of the air flow are fixed in the inlet of diffusers. The objective of this work is to identify the factors that may influence the efficiency of the ventilation performance, such as the jet configuration and the impact height. The studied configuration was numerically simulated by the finite volume method. The equations systems have been closed with the turbulence model $(k-\varepsilon)$. In general, the prediction of the flow field is in better agreement with the experimental data. The results obtained show that the studied parameters have significant effects on the flow characteristics and behavior of multi-jet impinging. The configuration of three jets with a $4 D$ impinging height has given a good development of the jet and an almost uniform temperature along the plate with the input temperature conditions in the diffusers $(T, T, T)$.
\end{abstract}

Key words: Swirling multi-jets / impacting a plate / thermal homogenization / ventilation

\section{Introduction}

The impinging jets are a very important practical interest in industry systems. As the drying fabric, the annealing, the cooling surfaces of the electronic parts or of the gas turbine blades, the quench glass and polymer processing, in order to optimize heat transfer between a fluid and a structure. They are commonly used in the industry for cooling process, heating or drying. Several experimental and numerical studies have shown the ability to improve the heat transfer through the implementation of the impact of jets. An aerodynamic and thermal experimental study of the impact of a single round jet on a plane plate was completed by Roux [1]. This study was conducted with a speaker that can modulate the velocity of the jet nozzle exit. The velocity measurements show that the forcing amplifies vortex structures in the jet mixing layer. According to the forcing, these vortices can be very stable and result in regular interactions between vortices. Parietal fluctuating temperature measurements reveal two propagation modes of temperature fluctuations on the impact plate. The first is independent of the acoustic forcig, and corresponds to a filament

\footnotetext{
${ }^{a}$ Corresponding author: zerroutamar@yahoo.fr
}

spread. The second is connected to the convection vortices on the impact plate and corresponds to the radial spread of cold and warm fronts. Another section aims to present the main general results on the aerodynamics of a round jet impact on a flat wall. We will limit ourselves to the case of jet impinging plate perpendicularly. Oguic et al. [2] Propose to characterize the heat exchanges occurring on the new generation of wind turbine rotors using numerical simulations DNS and LES guy with consideration of transfer by conduction in the rotor. The spatial discretization is done by compact schemes in order finite difference 4 for the radial and axial directions and Fourier series in the tangential direction. The treatment of the singularity at the axis is based on conditions of parity functions in Fourier space. The temporal discretization is semi-implicit order 2. To resolve the flow in the inlet nozzle and inter disk space and heat transfer in the rotor, a multi domain solver based on the technique of matrix influence was developed. To optimize the computing time, the solver has been parallelized using a hybrid parallelization Open MP/MPI. The accuracy of the numerical method has been verified and the code has been validated burst vortex type of test configurations in a cylindrical vessel, turbulent flows in a cylindrical pipe and simplified 


\section{Nomenclature}

\begin{tabular}{|ll|}
\hline$D$ & Nozzle diameter, $\mathrm{m}$ \\
$d$ & Support vane diameter, $\mathrm{m}$ \\
$G_{\theta}$ & Angular momentum flux, $\mathrm{kg} \cdot \mathrm{m}^{2} \cdot \mathrm{s}^{-2}$ \\
$G_{X}$ & Axial momentum flux, $\mathrm{kg} \cdot \mathrm{m}^{2} \cdot \mathrm{s}^{-2}$ \\
$H$ & Impacting height, $\mathrm{m}$ \\
$K$ & Kinetic energy of turbulence, $\mathrm{m}^{2} \cdot \mathrm{s}^{-2}$ \\
$R$ & Diffuser radius, $\mathrm{m}$ \\
$S$ & Swirl number \\
$R_{h}$ & Support vane diameter, $\mathrm{m}$ \\
$R_{n}$ & Diffuser radius, $\mathrm{m}$ \\
$R_{e}$ & Reynolds number \\
$T_{\mathrm{a}}$ & Ambient temperature, ${ }^{\circ} \mathrm{C}$ \\
$T_{i}$ & Jet temperature at the point considered, ${ }^{\circ} \mathrm{C}$ \\
$T_{\mathrm{m}}$ & Maximum temperature at the diffuser exit, ${ }^{\circ} \mathrm{C}$ \\
$T_{r}$ & Dimensionless temperature \\
$U$ & Axial mean velocity, m.s ${ }^{-1}$ \\
$U_{\mathrm{max}}$ & Maximum velocity at the diffuser exit, $\mathrm{m} . \mathrm{s}^{-1}$ \\
$U_{r}$ & Dimensionless axial velocity \\
$V$ & Radial velocity, m.s ${ }^{-1}$ \\
$W$ & Tangential velocity, m.s ${ }^{-1}$ \\
\hline & $\quad$ Greek symbols \\
\hline$\alpha$ & Vanes angle inclination \\
$C_{\mu}$ & Empirical constant \\
$\sigma t$ & Prandtl numbers for kinetic energy of turbulence \\
$\delta_{i j}$ & Tenso identity \\
$\varepsilon$ & The rate of dissipation of kinetic energy, $\mathrm{m}^{2} . \mathrm{s}^{-2}$ \\
$\mu_{t}$ & Turbulent viscosity, kg.m ${ }^{-1} \cdot \mathrm{s}^{-1}$ \\
$\rho$ & Density of air, kg.m ${ }^{-3}$ \\
\hline
\end{tabular}

stage turbocharger. For the case of jet impacting the geometrical parameters considered correspond to the experimental device, aspect ratio of the cavity $G=0.02$ and aspect ratio of the jet is 0.5 , The Reynolds number debiting the nozzle outlet is $R e=5300$. Three simulations are performed for three rotation parameters $N=0.0767$, 0.618 and 1.23 . This choice allows comparisons with measurements for hydrodynamic fields and thermal data and with predictions from such models RANS $k-\omega$ SST and RSM. Particular attention is paid to the influence of vortex structures in heat transfer mechanisms to the surface of the rotating disk. Many articles discuss this case, the main ones cited by.

Miller et al. [3] provided an additional information on the regions of the impinging jet of the structure by calling the respectively "impact area and transitional" and "wall jet region developed". It defines the characteristic quantities of the velocity profile in the wall jet, ie the maximum velocity $V_{\mathrm{m}}$, the local thickness b of the wall jet and the dimension $y_{1}=2$ where velocity is half $V_{\mathrm{m}}$ from the balance between the amount of movement of the jet and that of the wall jet (see Fig. 1).

\section{Experimental setup}

The experimental facility is presented in Figure 2. It consists of a chassis on which is fixed a square plexiglas plate $w(1)$. On the latter, three devices blowing hot air

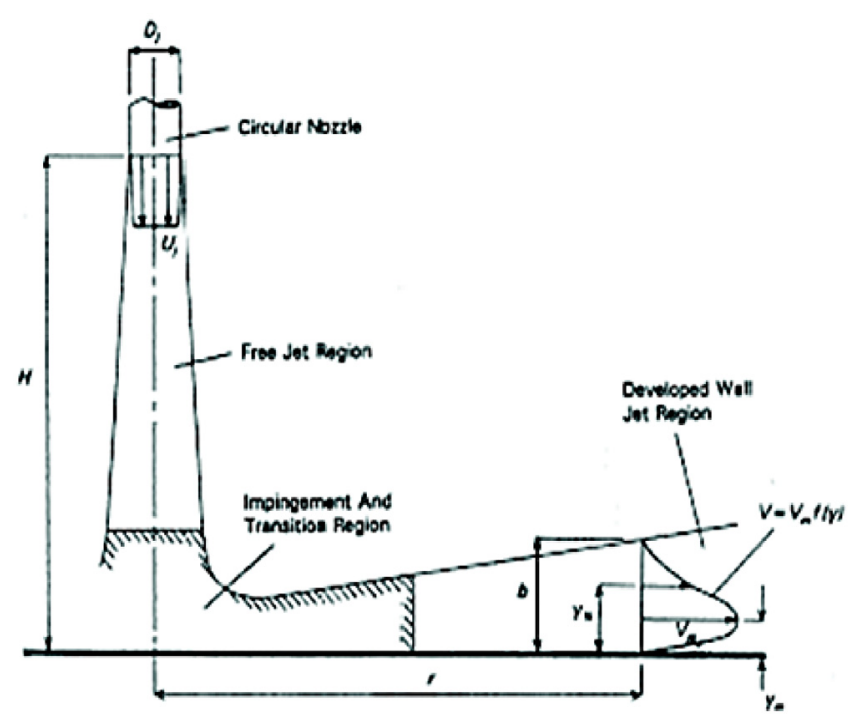

Fig. 1. Diagram definition of a single impinging jet and the radial wall jet, reproductive Miller [3].

are fixed and directed downwards, and the lower part of these devices is used to fix different types of diffusers provided with inclined vanes $\left(60^{\circ}\right)$, depending on the studied configuration (2). The ambient temperature $T_{\mathrm{a}}$ is measured at various points of the jet $T_{i}$ by a digital thermometer (3). The temperatures and velocity of the flow are measured by a thermo-anemometer (type Velocicalc Plus Air velocity Meter, Operation, and Service Manual 1980321 , Revision H., June 2006) which is a highprecision multifunctional instrument (4). The data can be viewed on screen, printed or downloaded to a spreadsheet program allowing us to easily transfer data to a computer for statistical treatment (5). The accuracy is of order $0.015 \mathrm{~m} . \mathrm{s}^{-1}$ for velocity and $0.3{ }^{\circ} \mathrm{C}$ for temperature from thermal sensor. Note that the thermal sensor is supported by rods which are easily guided vertically and horizontally to sweep the maximum space in the axial and radial directions (4). The blowing inlet temperature $T_{\max }$ is measured for each type and jet configuration (Fig. 2).

\section{The swirl number}

The existence of a azimuthal velocity component $(W)$ in the flow gives a rotation to flow fluid, which is marked by a swirl number $(S)$. This number is outlined as the ratio of the axial flux of tangential momentum to the product of the axial momentum flux and a characteristic radius [4]. It ought to be signaled that the exact expression of swirl number depends on the diffuser geometry and flow profiles. For a diffuser with a vane swirler, the definition of swirl number given by Gupta et al. [4] can be expressed as:

$$
S=G_{\theta} / R G_{x}=\int_{R_{n}}^{R_{h}} U W r^{2} d r / \int_{R_{n}}^{R_{h}} R_{n} U^{2} r \mathrm{~d} r .
$$




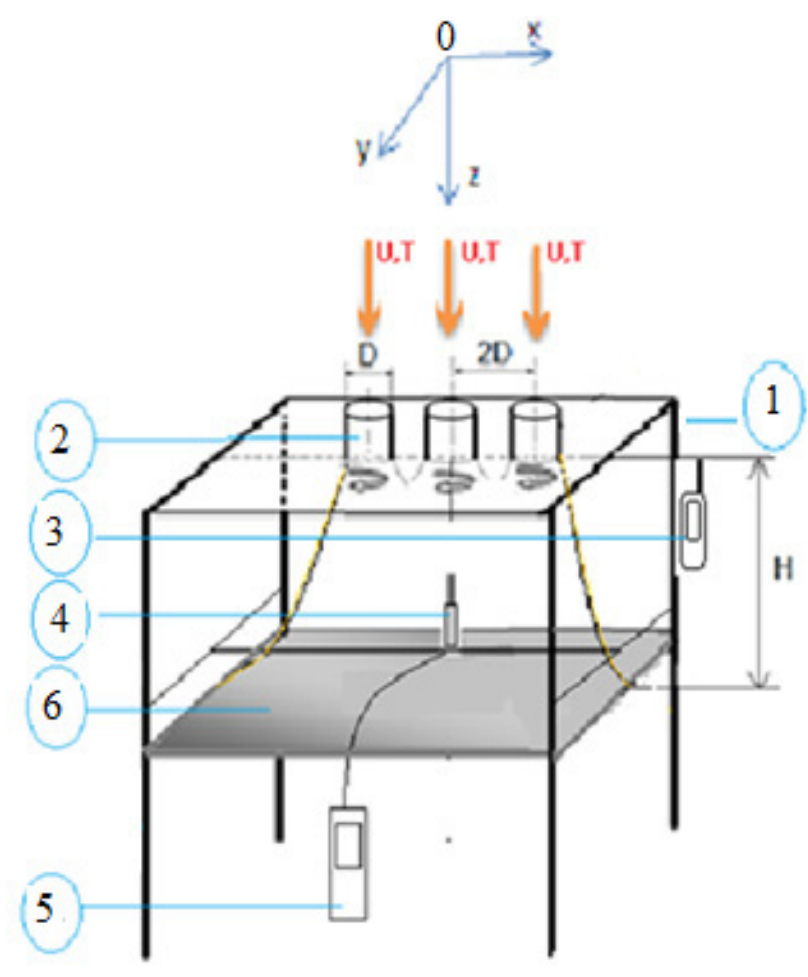

(a)

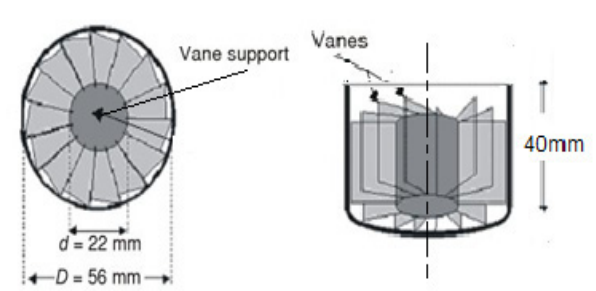

Cylindrical conduct
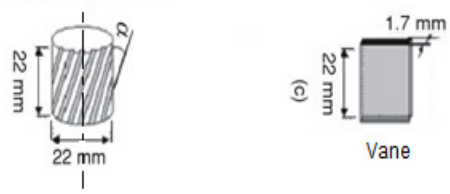

(b)

Fig. 2. Experimental configuration (a), Schematic of swirling diffuser (b).

The swirl number can be evaluated at any position of the jet because the two quantities are calculated. Swirling helps promote and improve the process of mixing and transfer, as well as the jet has the advantage of quickly flourish in free jets.

It ought to be noted here that if the axial and azimuthally velocities are assumed to be uniform and the vane are very thin, the swirl number can be expressed as:

$$
S=\frac{2}{3}\left[1-\left(\frac{R_{h}}{R_{n}}\right)^{3} / 1-\left(\frac{R_{h}}{R_{n}}\right)^{2}\right] \operatorname{tg} \alpha
$$

Such that:

$\alpha$ : is the angle of the fins built swirl generator

$R_{h}$ : is the radius of the vane diffuser support

$R_{n}$ : is the radius of diffuser

Note that in the case of a swirler without hub $\left(R_{h} \approx 0\right)$, the expression becomes [6]:

$$
S=\frac{2}{3} \operatorname{tg} \alpha
$$

In this study, the axial and azimuthal velocities $U$ and $W$, respectively, were measured at the outlet of a diffuser with a swirling jet hot wire anemometer triple probes (DISA55M01). Four values of the swirl number can be used, $S=0$ to $\alpha=0^{\circ}, S=0.4$ to $\alpha=30^{\circ}, S=0.7$ to $\alpha=45^{\circ}$ and $S=1.3$ to $\alpha=60^{\circ}$, respectively. The swirl number is used in this investigation, which corresponds to $\alpha=60^{\circ}, S=1.3[7]$.

\section{Numerical procedures}

For a steady, three-dimensional, incompressible, and turbulent flow with constant fluid properties, the governing equations of conservation of mass, momentum and energy are written in the cartesian tensor notation as follows [8]:

$$
\begin{aligned}
\frac{\partial U_{i}}{\partial x_{i}} & =0 \\
\rho \frac{\partial\left(U_{i} U_{j}\right)}{\partial x_{j}} & =-\frac{\partial P}{\partial x_{i}}+\frac{\partial}{\partial x_{j}}\left[\mu\left(\frac{\partial U_{i}}{\partial x_{j}}+\frac{\partial U_{j}}{\partial x_{i}}\right)-\overline{\rho u_{i}^{\prime} u_{j}^{\prime}}\right] \\
\rho C_{p} U_{i} \frac{\partial T}{\partial x_{i}} & =\frac{\partial}{\partial x_{i}}\left[\lambda \frac{\partial T}{\partial x_{i}}-\rho C_{p} \overline{u_{i}^{\prime} T^{\prime}}\right]
\end{aligned}
$$

where $U_{i}$ and $T$ denote the mean velocity and temperature; $u_{i}^{\prime}, u_{j}^{\prime}$ and $T^{\prime}$ are the corresponding fluctuation components; $-\rho \overline{u_{i}^{\prime} u_{i}^{\prime}}$ and $-\rho C_{p} \overline{u_{i}^{\prime} T^{\prime}}$ are the average Reynolds stresses and turbulent heat fluxes which need to be modeled to close the equations. It should be noted that here, the temperature variations are negligible and the Mach number is low $(<0.3)$, which allows us to assume that the fluid is incompressible (constant density).

The Boussinesq hypothesis, which relates the Reynolds stresses to the mean velocity gradients, is expressed as:

$$
-\rho \overline{u_{i}^{\prime} u_{j}^{\prime}}=\mu_{t}\left(\frac{\partial U_{i}}{\partial x_{j}}+\frac{\partial U_{j}}{\partial x_{i}}\right)-\frac{2}{3}\left(\rho k+\mu_{t} \frac{\partial U_{i}}{\partial x_{i}}\right) \delta_{i j}
$$


where $k$ is the turbulent kinetic energy, as defined by $k=\frac{1}{2} \overline{u_{i}^{\prime} u_{i}^{\prime}}$, and $\delta_{i j}$ is the tensor identity. An advantage of the Boussinesq approach is the relatively low computational cost associated with the computation of the turbulent viscosity $\mu_{t}$. Note that the turbulent viscosity $\mu_{t}$ is given by:

$$
\mu_{t}=C_{\mu} \rho k^{2} / \varepsilon
$$

Hence in the present calculation, the turbulence scalar fluxes are modeled using the gradient-diffusion approach [9] as:

$$
\rho C_{p} \overline{u_{i}^{\prime} T^{\prime}}=-\frac{C_{p} \mu_{t}}{\sigma_{t}} \frac{\partial T}{\partial x_{i}}
$$

where $\sigma t=0.6$ stands for the turbulent Prandtl number.

The $(k-\varepsilon)$ model is an example of two equation models that use the Boussinesq hypothesis. Here, two different closure models, $(k-\varepsilon)$ model and the Reynolds Stress Model (RSM), have been used.

\subsection{Reduced temperature}

The reduced temperature $\left(T_{r}\right)$ of measurement is obtained by reference to the maximum average temperature at the outlet of the blowing opening and at room temperature:

$$
T_{r}=\frac{T_{i}-T_{a}}{T_{\max }-T_{a}}
$$

\subsection{Reduced dimensionless axial velocity}

The reduced dimensionless axial velocity is obtained with respect to the maximum velocity at the outlet of the blowing port.

$$
U_{r}=\frac{U_{i}}{U_{\max }}
$$

Similarly, the radial and axial distances are given by reference to the diameter of the blowing diffuser in dimensionless form $r / R$ and $x / D$.

\subsection{Domain calculating mesh}

The computational domain is divided into a number of non-overlapping volumes called control volume such that each volume surrounding each point of the mesh. The calculation was made on an appropriate mesh with (865 421) tetrahedral structure of cells.

The differential equation is integrated to each volume control and the result of the integration gives the discrete equation expressed with the values of the function $\Phi$ (scalar quantity) for a set of grid points.

The obtained discrete equation expresses the conservation principle for $\Phi$ in the control volume in the same way that the differential equation expressed to a volume of infinitesimal control [10].

\section{Numerical results}

See Figures 4-7.

\section{Validation of results}

See Figure 8.

\section{Discussions}

\subsection{Profiles of reduced temperature}

The temperature profiles of these maxima (amplitudes) at the outputs of the first stations in diffusers of Figure 4. This decrease is due to the distance of the blowing diffuser and mixing of the jets. In the following amplitudes of the impoverished temperature from, a station to another, to reach the temperature at the downstream stations impact of the jet. Temperature curve becomes almost parallel to the obstacle, the impact is reduced amplitudes then radial ambient temperatures increase slightly and the overall development of a jet stations to another.

\subsection{Mean reduced axial velocity profiles}

In the beginning of this area the jet as a free jet, axial mean velocity profiles (Fig. 5), have amplitudes nival of each blow port, these amplitudes decrease from one station to another until the velocity curve becomes almost parallel to the baffle wall. In the presence of the impact the fluid particles change direction. The area of impact increases the vitality of the global jet over a free jet without impact.

These presented results show the decrease of the average axial velocity of the jet impact. This is largely due to the turbulence and the barrier effect produced by the producing lateral development of the jet while approaching the wall region.

\subsection{Temperature field and velocity field vectors}

Figures 6, 7, describes temperature field, and velocity field vectors to a height of impact $4 D$ and inlet temperatures $(T, T, T)$, we note that multi jet system is present at the start as a free jet is established the flow region from the injection port to the end of the cone potential then it is characterized by weakening of the velocity on the axis and development hence the established flow area, then the jet is deflected from its initial direction is axial deflection region. Finally, velocity is mainly radial and where the boundary layer, whose thickness increases radially, called the wall jet region. 


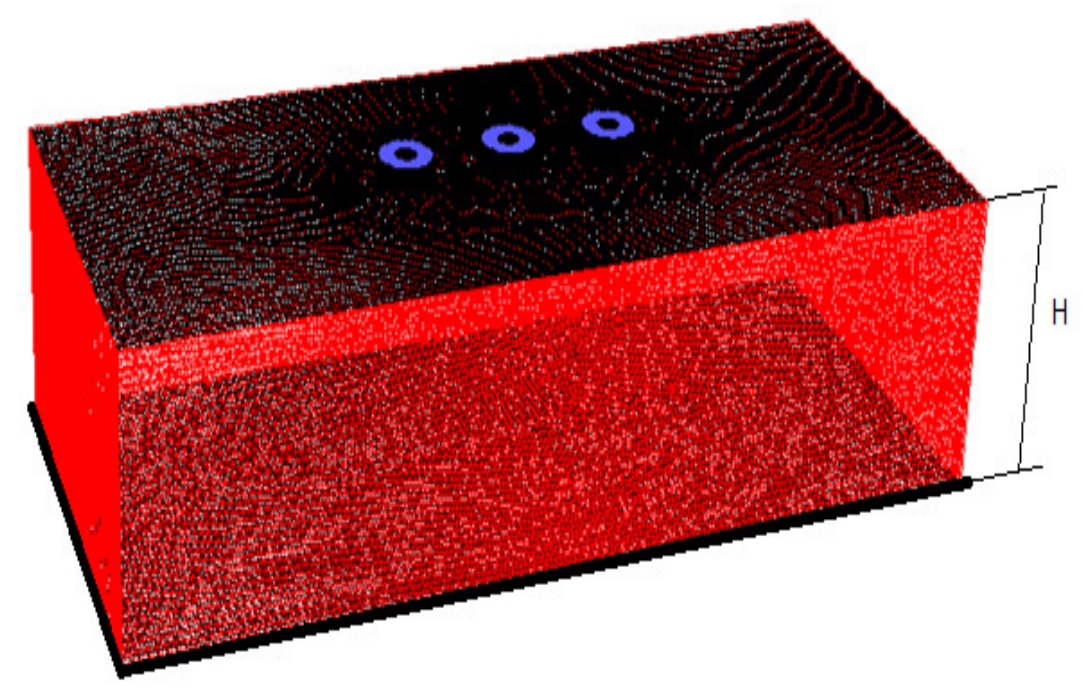

Fig. 3. Diagram of the mesh geometry 3 diffusers, height impact $4 D$.

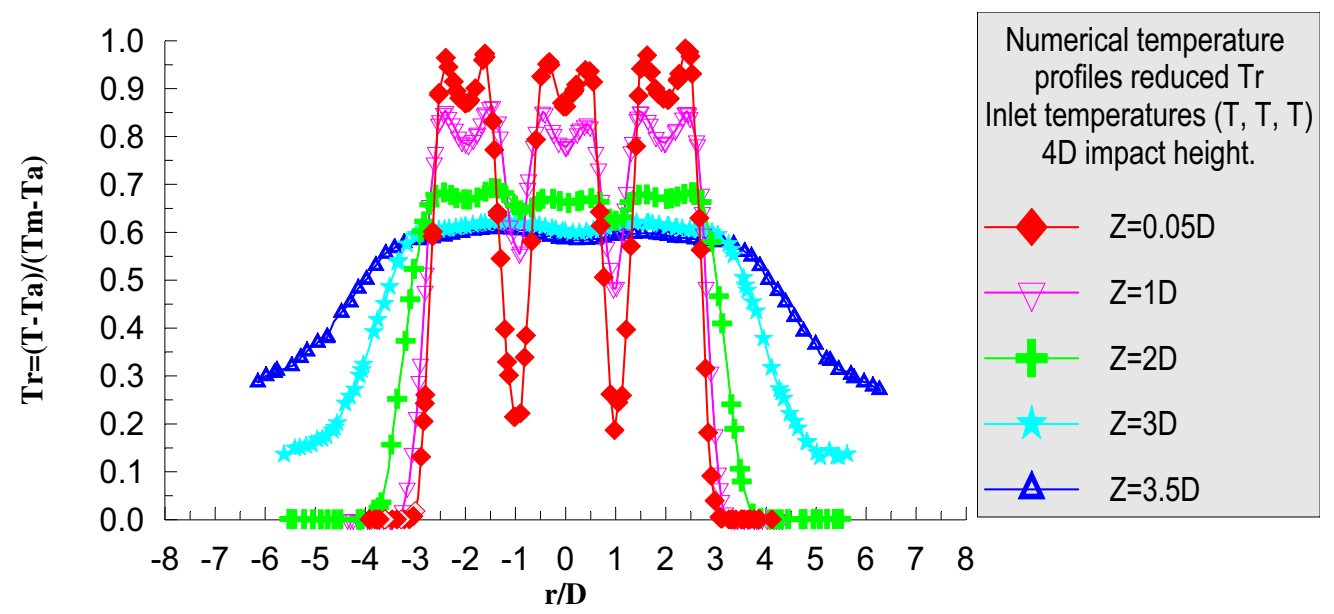

Fig. 4. Profiles of reduced temperature $T_{r}$ numerical model ( $k$-epsilon), height impact $4 D$, inlet temperature $(T, T, T)$.

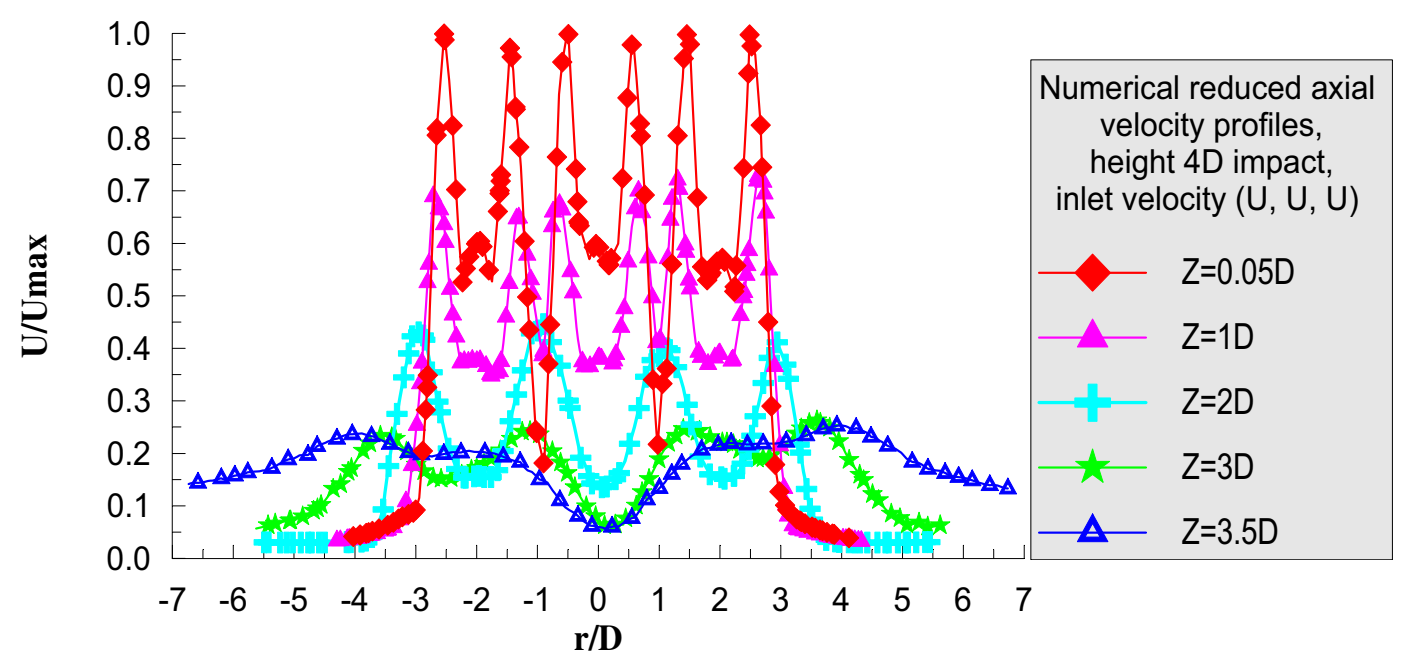

Fig. 5. Numerical velocity Profiles dimensionless $U / U_{\max }$ by model ( $k$-epsilon) height impact $4 D$, inlet velocities $(U, U, U)$. 


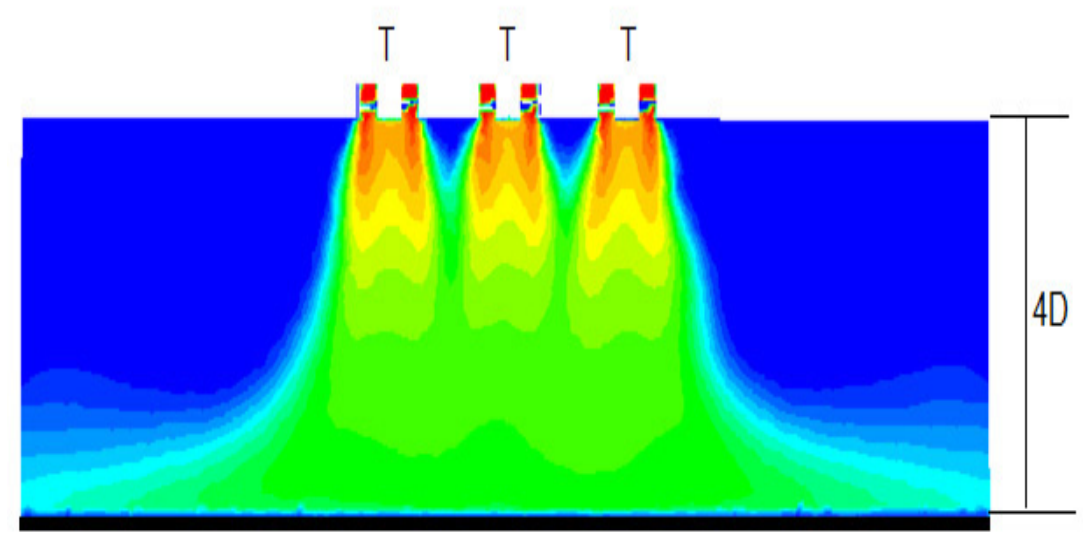

Fig. 6. Temperature field, height of impact $4 D$ inlet temperatures $(T, T, T)$.

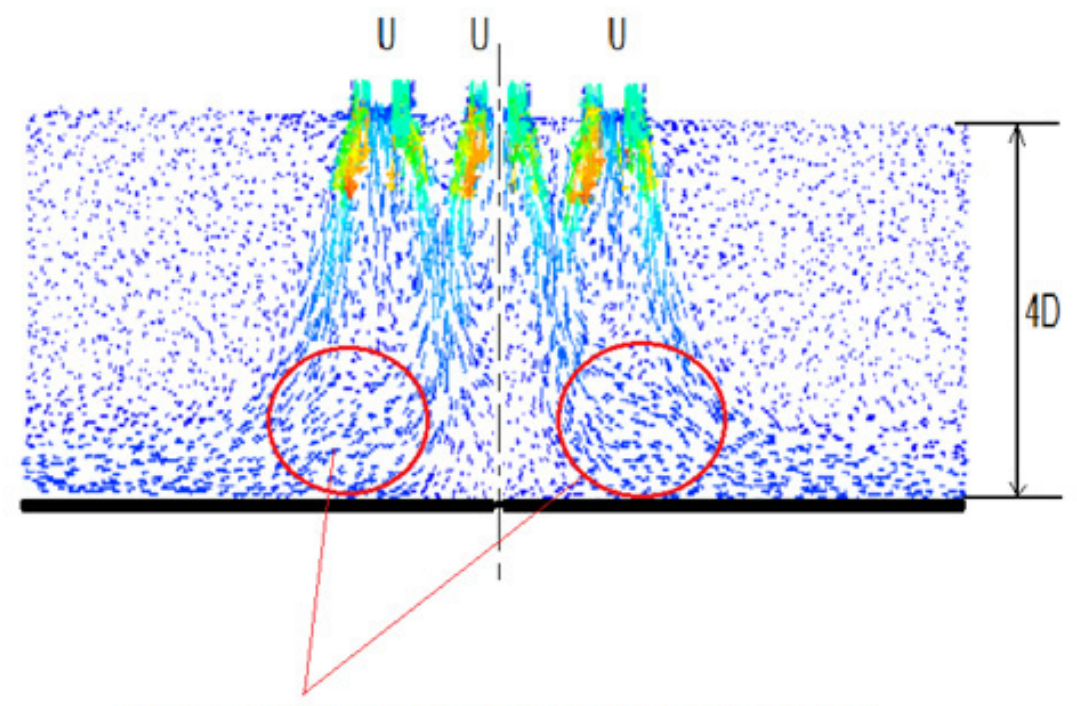

(Deflection Zone) the velocity vectors changes direction

Fig. 7. Velocity field vectors height of impact $4 D$ inlet velocities $(U, U, U)$.

\subsection{Validation of results}

Quality comparison between the numerical results and the experimental results is demonstrated in Figure 8, this comparison shows that the model with two transport equations $(k-\varepsilon)$ used to simulate this case produces results satisfactory. Despite the weaknesses of the model $(k-\varepsilon)$, the latter gave acceptable results qualitatively. Nevertheless, it is a relatively simple simulation tool inexpensive to use.

\section{Conclusion}

A numerical simulation of a multiple swirling jets system impacting a plane plate is presented above. The comparison shows that the simulation results agree with ex- perimental data very well. The studied configurations of swirling jet flow are as follows:

(1) Influence of impact plane height. The amplitude of the axial temperature close to the impact surface is greater when the height of impact decreases. Swirling provides thermal homogenization with a large spread. The fluid flow tend to move away towards the radial direction of the jet and slowed following the axial direction causing a large spreading.

(2) Influence of the jets position. Results presented clearly show that the first outboard jet has the same characteristics as a free jet, close to the obstacle it undergoes considerable deflection characterized by the weakening of velocity and vitality of the jet (amplitudes temperature decrease), in contact with the plate, the curves are almost parallel to the plate velocities change direction and expand radially. 

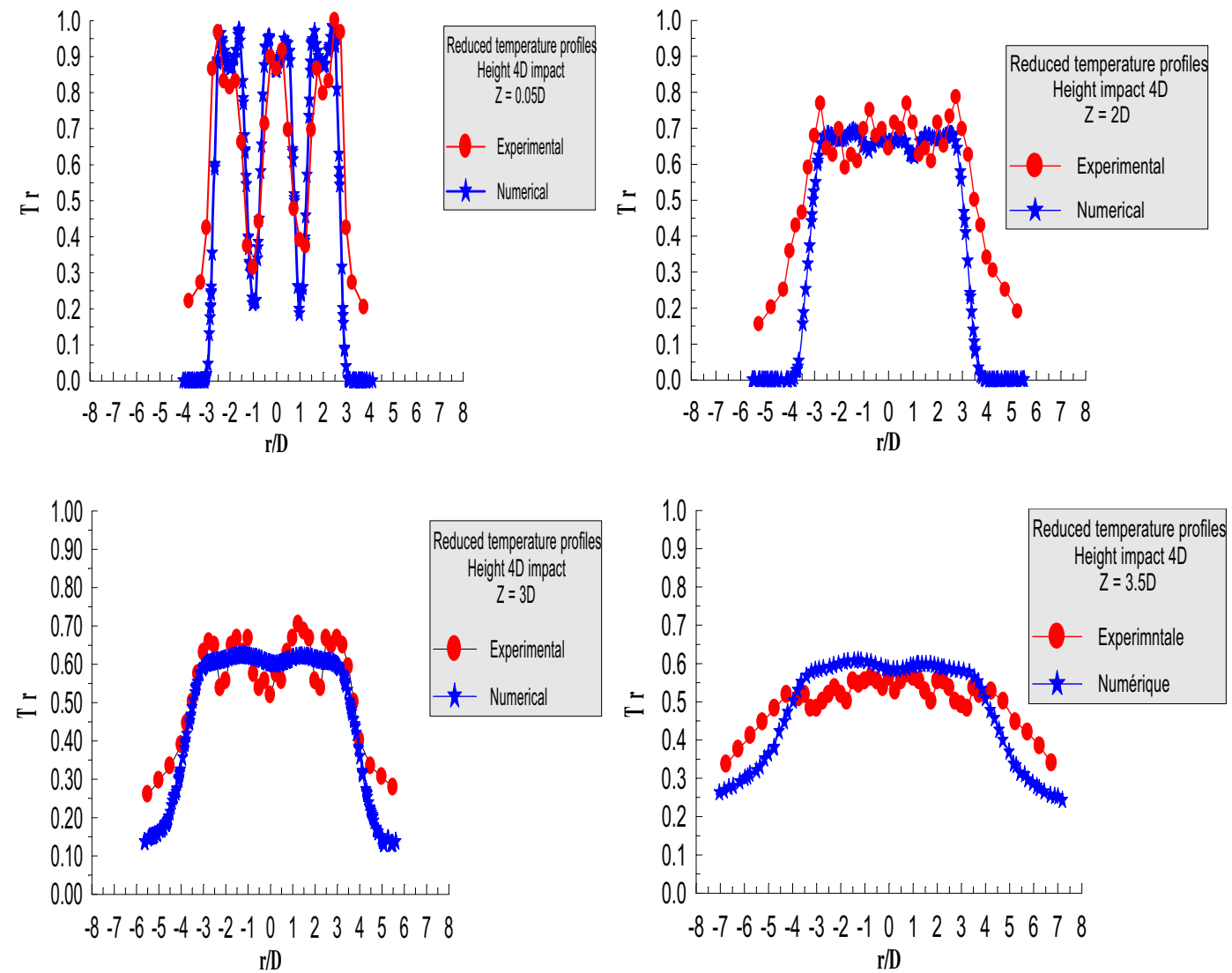

Fig. 8. Comparison of numerical temperature profiles and experimental, impact height $4 D$ inlet temperatures $(T, T, T)$.

(3) The comparison between the numerical results and the experimental results presented in this study indicates that the model with two transport equations $(K-\varepsilon)$ produced satisfactory results. Despite the weaknesses of the model $(K-\varepsilon)$, the latter gave acceptable results qualitatively. Nevertheless, it is a relatively simple simulation tool to study swirling multi-jet.

The presence of the plate reducing temperature amplitudes and the recirculation zone, the temperature of the plate decreases gradually as it moves away from the diffuser and becomes negligible beyond ten diameters of the orifice blowing. It can be said that a key objective in a configuration multi-jet swirling system is to access the perfect mix as soon as possible with a very high heat transfer plate; the rate of mixing can be influenced by other parameters, for example, sense of swirling of central jet etc. These will be investigated in our future study.

\section{References}

[1] S. Roux, Experimental contribution to the aerothermal a jet impact forced acoustically, Ph.D Thesis, University of Poitiers, National Engineering School of Poitiers, National Diploma-, Decree of 7 August 2006 Research: Fluid Mechanics-Thermal

[2] R. Oguic, S. Viazzo, S. Poncet, Numerical simulations of turbulent jet impact and heat transfer in a machine discoid, XII Interuniversity Symposium on Thermal Franco
Quebecers Systèmes, 8-10 of June, Sherbrook, Quebec, Canada, 2015

[3] P. Miller, A study of wall jets resulting from single and multiple inclined jets impingements, Aeronaut. J. (1995) 201-216

[4] A.K. Gupta, D.G. Lilley, N. Syred, Swirl flows, Abacus Press, London, 1984

[5] Y. Huang, V. Yang, Dynamics and Stability of LeanPremixed Swirl-Stabilized Combustion, Prog. Energy Combust. Sci. 35 (2009) 293-364

[6] H. Sato, M. Mori, T. Nakamura, Development of a Dry Ultra-low NOx Double Swirler Staged Gas Turbine Combustor, Energy Technology Research Institute, Tokyo [13]-Gas Co, Ldt Tokyo, Japan, J. Eng. 120 (1998)

[7] M. Braikia, A. Khelil, L. Loukarfi, H. Naji, Improvement of thermal homogenization using multiple swirling jets, Therm. Sci. 16 (2012) 239-250

[8] B.E. Launder, D.B. Spalding, The Numerical Computation of Turbulent Flows, Imperial Collogue of Science and Technology, Department of Mechanical Engineering Exhibition Road LONDON, received 13 august, 1973

[9] D.F.G. Durco, J.C.F. Pereira, Calculation of isothermal turbulent three dimensional free multijet flows, Institute Superior, Technical University of Lisbon, Lisbon, Portugal

[10] V.O. Dinh Ngoc, Numerical methods of Local Integral on Finite Volume, School of Engineering University Moncton, Canada

[11] FLUENT User's Guide, 2006 